\title{
Epidemiology and outcome of HIV-infected patients admitted to the ICU in the current highly active antiretroviral therapy era
}

\author{
Epidemiología y pronóstico de los pacientes con VIH ingresados en la UCI en la \\ era de tratamiento antirretroviral de gran actividad actual
}

\author{
P. Vidal-Cortés ${ }^{\mathrm{a}}$, L.A. Álvarez-Rocha ${ }^{\mathrm{b}}$, P. Fernández-Ugidos ${ }^{\mathrm{a}}$, M.A. Pérez- \\ Veloso $^{a}$, I.M. Suárez-Paul ${ }^{c}$, A. Virgós-Pedreira ${ }^{\mathrm{d}}$, S. Pértega-Díaz ${ }^{\mathrm{e}}$, Á.C. Castro- \\ Iglesias $^{\mathrm{f}}$
}

\footnotetext{
${ }^{a}$ Intensive Care Unit, Complexo Hospitalario Universitario de Ourense (CHUO), SERGAS, Spain

${ }^{b}$ Intensive Care Unit, Complexo Hospitalario Universitario de A Coruña (CHUAC), SERGAS, Spain

${ }^{c}$ Intensive Care Unit, Hospital San Juan de Dios, Córdoba, Spain

${ }^{d}$ Intensive Care Unit, Complexo Hospitalario Universitario de Santiago de Compostela (CHUS), SERGAS, Spain

${ }^{e}$ Clinical Epidemiology and Biostatistics Research Group, Instituto de Investigación Biomédica de A Coruña (INIBIC), Complexo Hospitalario Universitario de A Coruña (CHUAC), SERGAS, Universidade de A Coruña, Spain

${ }^{f}$ Grupo de Virología Clínica, Instituto de Investigación Biomédica de A Coruña (INBIC) - Complexo Hospitalario Universitario de A Coruña (CHUAC), SERGAS, Universidade de A Coruña, Spain
}

\begin{abstract}
Purpose. To describe the epidemiology of critical disease in HIV-infected patients during the current highly active antiretroviral therapy (HAART) era and to identify hospital mortality predictors.

Methods. A longitudinal, retrospective observational study was made of HIV-infected adults admitted to the ICU in two Spanish hospitals between 1 January 2000 and 31 December 2014. Demographic and HIV-related variables were analyzed, together with comorbidities, severity scores, reasons for admission and need for organ support. The chi-squared test was used to compare categorical variables, while continuous variables were contrasted with the Student's $t$-test, Mann-Whitney $U$-test or Kruskal-Wallis test, assuming an alpha level $=0.05$. Multivariate logistic regression analysis was used to calculate odds ratios for assessing correlations to mortality during hospital stay. Joinpoint regression analysis was used to study mortality trends over time.

Results. A total of 283 episodes were included for analyses. Hospital mortality was $32.9 \%$ (95\%CI: $21.2-$ 38.5). Only admission from a site other than the Emergency Care Department (OR 3.64, 95\%CI: 1.30$10.20 ; p=0.01$ ), moderate-severe liver disease (OR 5.65, 95\%CI: $1.11-28.87 ; p=0.04$ ) and the APACHE II score (OR 1.14, 95\%CI: 1.04-1.26; $p<0.01$ ) and SOFA score at $72 \mathrm{~h}(\mathrm{OR} 1.19,95 \% \mathrm{CI}: 1.02-1.40 ; p=0.03$ ) maintained a statistically significant relationship with hospital mortality.

Conclusions. Delayed ICU admission, comorbidities and the severity of critical illness determine the prognosis of HIV-infected patients admitted to the ICU. Based on these data, HIV-infected patients should receive the same level of care as non-HIV-infected patients, regardless of their immunological or nutritional condition.
\end{abstract}




\begin{abstract}
Resumen
Objetivos. Describir la epidemiología de la patología crítica en el paciente infectado por VIH durante la era de tratamiento antirretroviral de gran actividad actual y encontrar predictores de mortalidad hospitalaria.

Métodos. Estudio observacional, retrospectivo y longitudinal que incluye pacientes infectados por VIH adultos ingresados en las UCI de hospitales de Galicia, entre el 1 de enero de 2000 y el 31 de diciembre de 2014. Analizamos variables demográficas y relacionadas con la infección por el VIH, comorbilidades, puntuaciones de gravedad, motivo de ingreso y necesidad de soporte de órganos. Empleamos la prueba de la Chi-cuadrado para comparar las variables categóricas y las pruebas de la T-Student, U de Mann-Whitney o H de Kruskal-Wallis para las variables continuas, asumiendo un error $\alpha=0,05$. Utilizamos la regresión logística multivariante para calcular la OR de la asociación con la mortalidad hospitalaria. Aplicamos análisis de regresión de joinpoint para estudiar la tendencia temporal de mortalidad.

Resultados. Incluimos 283 episodios. La mortalidad hospitalaria fue del 32,9\% (IC 95\% = 21,2-38,5\%). El ingreso no procedente de Urgencias (OR 3,64; IC 95\% $=1,30-10,20 ; \mathrm{p}=0,01)$, la enfermedad hepática moderada-grave (OR 5,65; IC 95\% =1,11-28,87; $\mathrm{p}=0,04)$ ), el APACHE II (OR 1,14; IC 95\% =1,04-1,26; $\mathrm{p}<0,01)$ y el SOFA a las $72 \mathrm{~h}(\mathrm{OR} 1,19$; IC 95\% = 1,02-1,40; $\mathrm{p}=0,03)$ se relacionan con la mortalidad hospitalaria en el análisis multivariante.

Conclusiones. El retraso en el ingreso en UCI, las comorbilidades y la gravedad del episodio determinan el pronóstico del paciente infectado por VIH ingresado en UCI. Los pacientes con VIH deberían recibir el mismo nivel de cuidados que los pacientes no infectados por VIH, independientemente de su estado inmunológico o nutricional.
\end{abstract}

\title{
Keywords
}

HIV; HAART; Prognosis

Palabras clave

VIH; TARGA; Pronóstico

\section{Introduction}

The introduction of antiretroviral therapy (ART) has meant a drastic change in HIV-infected patients' prognoses. Since its introduction, survival rates have continued to increase, even in developing countries. ${ }^{1,2,3}$ Moreover, high activity ART (HAART) has reduced the incidence of AIDS-related diseases and hospitalizations. ${ }^{4}$ Among patients with good immunovirological conditions, HIV infection has become a chronic disease. However, immune system restoration is incomplete in these patients and there remains an inflammatory condition which, in the long-term, is related to pathologies such as cancer and cardiovascular diseases. This chronic inflammatory profile, along with longer survival and possible toxicity accumulation from HAART, will mean that HIV-infected elderly patients have a high burden of "special" comorbidities known as HIVassociated non-AIDS (HANA) ${ }^{5}$ which will cause their hospital admissions.

HAART has also led to a significant change in both the profile and prognosis of HIV patients admitted to intensive care units (ICU). At the start of the pandemic in the early 1980 s, $70 \%$ of admissions were due to respiratory failure secondary to pneumonia caused by Pneumocystis jiroveci (formerly known as Pneumocystis carinii, hence its acronym: PCP). However, since HAART was introduced, the rate of patients admitted to the ICU with AIDS-related diseases has dropped, as has the associated mortality. In Europe and North America, short-term mortality of approximately 20-30\% (survival higher than $90 \%$ in the ICU) has been reported, as has survival of $85 \%$ at one year and higher than $70 \%$ at two years. $6,7,8,9,10,11,12,13,14,15,16,17,18,19,20,21,22,23$ Although with higher mortality than in Europe and North America, decreased mortality has also been observed in countries such as Brazil, Mexico, China, Taiwan and Australia, where intra-ICU and hospital mortality varies from $37 \%$ to $68 \%$ and $48 \%$ to $68 \%$, respectively. ${ }^{24,25,26,27,28,29,30}$ In Spain, higher mortality has been published compared with other developed countries $(36.5 \%, 67.9 \%$ and $80.9 \%$ at 30,60 and 90 days, respectively $)^{31}$ without evidence of a decrease over the HAART period. ${ }^{32}$ 
However, despite the decreased mortality in HIV patients admitted to the ICU, this is still greater than in non-HIV patients. ${ }^{20,23,31}$ Results from analyses of prognostic factors in this patient group have varied. Some study authors have concluded that the patient's immunovirological condition determines the prognosis, ${ }^{26,28}$ while others have stated that prognosis depends on disease severity that led to ICU admission ${ }^{19,21,22,25,27,33}$ and comorbidities. ${ }^{21,22,31}$

Our study aim was thus to describe the epidemiology of critical pathology in HIV patients in a cohort of patients admitted to ICU, during the current HAART period and to identify factors that predict mortality during hospital admission.

\section{Methods}

\section{Study scope}

The study was performed in the ICUs of Complexo Hospitalario Universitario de A Coruña (36 beds) and Complexo Hospitalario Universitario de Ourense (22 beds), two public hospitals located in the northwest of Spain, serving an approximate population of 500,000 inhabitants.

\section{Study period}

Data were collected from 1 January 2000 to 31 December 2014.

\section{Study design}

This was a longitudinal, retrospective, observational study.

\section{Inclusion criteria}

Inclusion criteria included: ICU admission from the community, hospital ward or another hospital; HIV infection diagnosed previously or during ICU admission; age older than 18 years.

\section{Exclusion criteria}

Patients readmitted to the ICU during the same hospital admission were excluded.

\section{Patient recruitment}

Patients were identified for inclusion using the two hospitals' clinical documentation systems.

\section{Ethics}

Ethical approval was granted by the Pontevedra/Vigo/Ourense Research Ethics Committee (code 2015/489). 


\section{Measurements}

Clinical records for each admission were reviewed. We analyzed sociodemographic data (age and sex); comorbidities (defined according to the Charlson comorbidity index - CCI, see Appendix A. Supplementary data); date of HIV infection diagnosis; most recent CD4 count and viral load (VL) (in the event that these data were unavailable for the six months prior to admission, we used the first post-admission data if determined during the first week of ICU admission); HAART (active prescription of at least three antiretrovirals from at least two classes, unless there was evidence of therapeutic non-compliance); nutritional status (cachexia, reported during physical examination, and albumin levels upon ICU admission); reason for ICU admission; severity at admission (APACHE II score); organ dysfunction (SOFA score at admission and $72 \mathrm{~h}$ ); organ support during admission (vasopressors, mechanical ventilation and renal replacement techniques [RRTs]); ICU/hospital length of stay (LOS) and ICU and in-hospital mortality. We also recorded ART administration during the ICU stay.

Admission from ED (Emergency Department) was defined as ICU admission right away from ED. Admission form medical or surgical ward was defined as "admission not from E.D."

Patients with new positive tests during hospital admission and before discharge from the ICU were considered newly diagnosed with an HIV infection.

Co-infection with Hepatitis C Virus (HCV) was defined as positive RNA-HCV and Hepatitis B Virus infection (HBV) was defined as positive surface antigen (HBsAg).

To identify changes over time, we established three periods of 5 years (1: 2000-2004, 2: 20052009, 3: 2010-2014).

\section{Sample size rationale}

A total of 283 patients were recruited during the study period. This sample size allows estimating parameters with a precision of $\pm 6.5 \%$ at a confidence level of $95 \%$. Furthermore, it allows to detect as statistically significant differences in hospital mortality rates of $16 \%$ or higher, assuming a mortality rate around $25 \%$ and an expected exposure to a risk factor of $50 \%$ (confidence level $95 \%$, power level $80 \%$ ).

\section{Statistical analysis}

Continuous variables are shown as mean \pm standard deviation (SD) or median and p25-p75; qualitative variables are shown as number and percentage.

We compared patients who survived hospital admission with those who died during their hospital stay using the Chi-square test for categorical variables and Student's $t$-test or the MannWhitney $U$ test for continuous variables, assuming an alpha level $=0.05$. We compared the three time periods using Kruskal-Wallis $H$ for quantitative variables, and Chi-square for categorical variables. Trends in mortality during the study period were investigated by a joinpoint regression analysis. We used logistic regression to calculate OR values to evaluate the association with mortality during the hospital stay. Variables associated with hospital mortality $(p<0.05)$ on univariate analyses were included in the multivariate analysis.

Statistical analyses were performed using IBM SPSS Statistics, Version 19.0. (IBM Corp., Armonk, NY). 


\section{Results}

A total of 297 admissions were recorded; among these, 14 patients were admitted twice, so only the initial 283 episodes were included for final analyses. The patient sample characteristics are shown in Table 1.

Table 1. Baseline characteristics of patients.

\begin{tabular}{|c|c|c|c|c|}
\hline & \multirow{3}{*}{$\begin{array}{c}\text { All } \\
n=283\end{array}$} & \multicolumn{2}{|c|}{ Hospital mortality } & \multirow{3}{*}{$p$ value } \\
\hline & & \multirow{2}{*}{$\begin{array}{c}\text { No } \\
190(67.1)\end{array}$} & \multirow{2}{*}{$\begin{array}{c}\text { Yes } \\
93(32.9)\end{array}$} & \\
\hline & & & & \\
\hline Age (years) & $43.34 \pm 9.60$ & $43.20 \pm 9.67$ & $43.63 \pm 9.53$ & 0.73 \\
\hline Male sex & $206(72.8)$ & $132(69.5)$ & $74(79.5)$ & 0.08 \\
\hline Admission from ED & $160(56.5)$ & $128(67.3)$ & $32(34.4)$ & $<0.01$ \\
\hline \multicolumn{5}{|l|}{ HIV infection } \\
\hline Diagnosis at this admission & $33(11.7)$ & $16(8.4)$ & $17(18.2)$ & 0.02 \\
\hline Diagnosis in the ICU & $23(8.1)$ & $13(6.8)$ & $10(10.7)$ & 0.26 \\
\hline HAART & $155(54.8)$ & $112(58.9)$ & $43(46.2)$ & 0.04 \\
\hline HAART in the ICU & $111(39.2)$ & $87(45.8)$ & $24(25.8)$ & $<0.01$ \\
\hline OI prophylaxis & $41(14.5)$ & $23(12.1)$ & $18(19.3)$ & 0.10 \\
\hline AIDS & $195(68.9)$ & $124(65.2)$ & $71(76.3)$ & 0.06 \\
\hline Years of infection & $9(3,16)$ & $10(5,16)$ & $8(1,16)$ & 0.10 \\
\hline CD4 count (cell/mL) & $167.0(53.5,414.0)$ & $204.0(74.0,493.5)$ & $80.0(29.0,236.5)$ & $<0.01$ \\
\hline Viral load (log) & $2.9(0.0,5.3)$ & $2.6(0.0,5.0)$ & $4.5(1.1,5.5)$ & 0.02 \\
\hline Viral load suppressed & $64(22.6)$ & $47(29.7)$ & $17(24.3)$ & 0.40 \\
\hline \multicolumn{5}{|c|}{ Comorbidities and nutritional status (see. Appendix A. Supplementary data) } \\
\hline DM free of complications & $10(3.5)$ & $5(2.6)$ & $5(5.4)$ & 0.24 \\
\hline DM with complications & $2(0.7)$ & $2(1.0)$ & $0(0)$ & 0.32 \\
\hline Cardiac disease & $13(4.6)$ & $10(5.2)$ & $3(3.2)$ & 0.44 \\
\hline Lung disease & $25(8.8)$ & $20(10.5)$ & $5(5.3)$ & 0.15 \\
\hline Liver disease & $185(65.4)$ & $122(64.2)$ & $63(67.7)$ & 0.56 \\
\hline M-S liver disease & $37(13.1)$ & $18(9.4)$ & $19(20.4)$ & 0.01 \\
\hline Renal disease & $5(1.8)$ & $2(1.0)$ & $3(3.2)$ & 0.19 \\
\hline Hepatitis C Virus (HCV) & $170(60.1)$ & $114(60.0)$ & $56(60.2)$ & 0.97 \\
\hline Hepatitis B Virus (HBV) & $36(12.7)$ & $27(14.2)$ & $9(9.7)$ & 0.28 \\
\hline $\mathrm{HCV}+\mathrm{HBV}$ & $24(8.5)$ & $20(10.5)$ & $4(4.3)$ & 0.08 \\
\hline Charlson Comorbidity Index & $7(3,7.25)$ & $6(2,7)$ & $7(6,8)$ & $<0.01$ \\
\hline Cachexia & $70(24.7)$ & $35(18.4)$ & $35(37.6)$ & $<0.01$ \\
\hline Albumin (g/dL) & $2.6(2.1,3.3)$ & $2.7(2.2,3.3)$ & $2.3(1.8,3.0)$ & $<0.01$ \\
\hline \multicolumn{5}{|c|}{ Diagnosis at admission (see. Appendix A. Supplementary data) } \\
\hline Severe sepsis/septic shock & $140(49.5)$ & $83(43.7)$ & $57(61.3)$ & $<0.01$ \\
\hline Cardiac & $55(19.4)$ & $48(25.2)$ & $7(7.5)$ & $<0.01$ \\
\hline Trauma & $19(6.7)$ & $15(7.9)$ & $4(4.3)$ & 0.26 \\
\hline Neurological & $11(3.9)$ & $4(2.1)$ & $7(7.5)$ & 0.04 \\
\hline \multicolumn{5}{|l|}{ Organ support during admission } \\
\hline Mechanical ventilation & $172(60.8)$ & $91(47.9)$ & $81(87.1)$ & $<0.01$ \\
\hline Vasoactive drugs & $114(40.3)$ & $55(28.9)$ & $59(63.4)$ & $<0.01$ \\
\hline Renal replacement therapy & $31(11.0)$ & $9(4.7)$ & $22(23.6)$ & $<0.01$ \\
\hline \multicolumn{5}{|l|}{ Complications } \\
\hline Superinfection & $41(14.5)$ & $19(10.0)$ & $22(23.6)$ & $<0.01$ \\
\hline \multicolumn{5}{|l|}{ Severity scores } \\
\hline APACHE II & $18.05 \pm 9.05$ & $14.90 \pm 7.97$ & $24.41 \pm 7.69$ & $<0.01$ \\
\hline SOFA score & $6(3,9)$ & $4(2,8)$ & $9(5,12)$ & $<0.01$ \\
\hline SOFA score $72 \mathrm{~h}$ & $5(2,8)$ & $3(2,7)$ & $7(4,11)$ & $<0.01$ \\
\hline SOFA $0-72 \mathrm{~h}$ & $-1(-3,1)$ & $-1(-3,0)$ & $0(-2,3)$ & $<0.01$ \\
\hline
\end{tabular}

Numbers reported as $\mathrm{n}(\%)$ and mean \pm standard deviation or median $(\mathrm{p} 25-\mathrm{p} 75)$

ED: Emergency Department; HAART: high-activity antiretroviral therapy; OI: opportunistic infection; DM: diabetes mellitus; M-S liver disease: moderate-severe liver disease. 
A total of 71 patients $(25.1 \%)$ died during their admission to the ICU, while another 22 died before hospital discharge, translating to a total hospital mortality of $32.9 \%$ (95\% CI $=21.2-38.5 \%$ ). Among these 14 patients who were admitted twice, 11 (78.6\%) died during hospital stay.

Median ICU and hospital LOS were $5^{2,13}$ and $18^{9,37}$ days, respectively.

In this sample, $68.9 \%$ fulfilled the AIDS criteria following CDC 1993 criteria. ${ }^{34}$ Following new definitions ${ }^{35} 11.7 \%$ patients were in stage $0,12.0 \%$ in stage $1,14.1 \%$ in stage $2,59 \%$ in stage 3 and 3.2 in stage unknown. Among patients with a known HIV infection, 62\% were under HAART at the time of admission (54.8\% of the total sample). Their CD4 count was 167.0 $(53.5,414.0)$ cells $/ \mathrm{mm}^{3}$ and VL was $\log 2.9(0.0,5.3)$, with $22.6 \%$ having an undetectable VL. Patients who survived their hospital admission, compared with those who died, received ART more frequently (58.9 compared with $46.2 \%, p<0.01)$ and had a better immunovirological profile: higher CD4 count $(204.0(74.0,493.5)$ cell $/ \mathrm{mL}$ compared with $80.0(29.0,236.5), p<0.01)$, and lower VL $(2.6(0.0,5.0) \log$ compared with $4.5(1.1,5.5), p=0.02)$.

The most common comorbidity in this sample was liver disease (present in $65.4 \%$ ), with $60.1 \%$ co-infected with HCV (Appendix A. Supplementary data, Table e1). Their CCI (median, IQR) was $6^{2,7}$ points. Both moderate-severe liver disease and CCI were statistically higher in patients who died in the hospital. A total of $24.7 \%$ of all patients were reported as cachectic upon physical examination, with median albumin $2.6(2.1,3.3) \mathrm{g} / \mathrm{dL}$ and a statistically high proportion of cachectic patients and lower albumin figures among those who did not survive.

The most common cause for admission was severe sepsis/septic shock (SS/SS) (49.5\%), followed by admissions with a cardiologic origin (19.4\%) (Appendix A. Supplementary data, Table e2). $61.3 \%$ of patients who died had been admitted due to SS/SS.

APACHE II scores upon admission and SOFA scores during the first $24 \mathrm{~h}$ were $18.05 \pm 9.05$ and $6,{ }^{3,9}$ respectively. A total of $60.8 \%$ of patients required respiratory support with invasive mechanical ventilation (MV) (length of $\mathrm{MV}: 7$ days $[2,13.75]$ ); $40.3 \%$ received vasoactive drugs (VADs) (length of VADs: 2 [1, 4] days), and 11\% required RTTs $(5$ [2, 11] days). Patients who died in the hospital presented significantly higher severity scores at admission and required more frequent respiratory, haemodynamic and renal support.

Comparison between the three periods is shown in Table 2. During the study period, the figures for hospital mortality did not vary significantly, being 30.3\% among patients admitted in 2000-2004, $31.8 \%$ in $2005-2009$ and $36.4 \%$ in 2010-2014 (annual percentage of change $=1.6 ; 95 \% \mathrm{CI}-2.3$ to $5.8, p=0.39)$. 
Table 2. Comparison based on year of admission.

\begin{tabular}{|c|c|c|c|c|}
\hline & $\begin{array}{c}2000-2004 \\
(n=66)\end{array}$ & $\begin{array}{c}2005-2009 \\
(n=129)\end{array}$ & $\begin{array}{c}2010-2014 \\
(n=88)\end{array}$ & $p$ value \\
\hline Age & $40.41 \pm 10.40$ & $42.27 \pm 8.66$ & $47.22 \pm 9.18$ & $<0.01$ \\
\hline \multicolumn{5}{|l|}{ HIV infection } \\
\hline New diagnosis & $7(10.6)$ & $10(7.8)$ & $16(18.2)$ & 0.06 \\
\hline Years of infection & $6(1,11)$ & $10(5,16)$ & $12(1.25,19)$ & $<0.01$ \\
\hline AIDS & $45(68.2)$ & $102(79.1)$ & $48(54.5)$ & $<0.01$ \\
\hline CD4 count (cell/mL) & $178.5(54,382.5)$ & $153.5(63.5,400)$ & $179.0(45,465)$ & 0.91 \\
\hline Viral load & $4.4(2.60,5.37)$ & $3.3(0,5.3)$ & $1.61(0,4.97)$ & $<0.01$ \\
\hline Viral load suppressed & $5(10.4)$ & $27(26.0)$ & $32(42.1)$ & $<0.01$ \\
\hline HAART & $26(39.4)$ & $72(55.8)$ & $57(64.8)$ & $<0.01$ \\
\hline HAART in ICU & $15(22.7)$ & $46(35.7)$ & $50(56.8)$ & $<0.01$ \\
\hline \multicolumn{5}{|l|}{ Comorbidities } \\
\hline M-S liver disease & $3(4.5)$ & $20(15.5)$ & $14(15.9)$ & 0.06 \\
\hline Charlson Comorbidity Index & $6.50(2,7)$ & $7(6,8)$ & $6(1.25,7.75)$ & $<0.01$ \\
\hline \multicolumn{5}{|l|}{ Diagnosis at admission } \\
\hline SS/SS & $27(40.9)$ & $70(54.3)$ & $43(48.9)$ & 0.21 \\
\hline PCP & $10(15.2)$ & $7(5.4)$ & $8(9.1)$ & 0.07 \\
\hline Cardiac & $19(28.8)$ & $19(14.7)$ & $17(19.3)$ & 0.06 \\
\hline Trauma & $6(9.1)$ & $6(4.7)$ & $7(8.0)$ & 0.43 \\
\hline Neurological & $2(3.0)$ & $3(2.3)$ & $6(6.9)$ & 0.22 \\
\hline \multicolumn{5}{|l|}{ Organ support } \\
\hline Mechanical ventilation & $37(56.1)$ & $75(58.1)$ & $60(68.2)$ & 0.22 \\
\hline Vasoactive drugs & $19(28.8)$ & $57(44.2)$ & $38(43.2)$ & 0.09 \\
\hline Renal replacement therapy & $4(6.1)$ & $13(10.1)$ & $14(15.9)$ & 0.14 \\
\hline \multicolumn{5}{|l|}{ Severity scores } \\
\hline APACHE II & $14(9,20.50)$ & $18(12,26)$ & $20(13,26)$ & $<0.01$ \\
\hline SOFA score & $4(1.25,7)$ & $7(3,11)$ & $7(3,9)$ & $<0.01$ \\
\hline SOFA score $72 \mathrm{~h}$ & $3(1,6)$ & $4(2,7.75)$ & $6(2,10)$ & $<0.01$ \\
\hline SOFA $0-72 \mathrm{~h}$ & $0(-1,0)$ & $0(-1,0)$ & $0(-1,0)$ & $<0.01$ \\
\hline \multicolumn{5}{|l|}{ Length of stay } \\
\hline ICU & $4(1.75,15)$ & $5(2,13)$ & $5.5(2,11)$ & 0.73 \\
\hline Hospital & $17(8,31)$ & $20(9,38)$ & $16(8,42.25)$ & 0.78 \\
\hline \multicolumn{5}{|l|}{ Mortality } \\
\hline ICU mortality & $17(25.8)$ & $30(23.3)$ & $24(27.3)$ & 0.79 \\
\hline Hospital mortality & $20(30.3)$ & $41(31.8 \%)$ & $32(36.4)$ & 0.69 \\
\hline
\end{tabular}

Numbers reported as $n(\%)$ and mean \pm standard deviation or median (interquartile range).

HAART: high-activity antiretroviral therapy; $\mathrm{M}-\mathrm{S}$ liver disease: moderate-severe liver disease, PCP: Pneumocystis jiroveci pneumonia.

In Table 3 we present differences between patients who were receiving HAART at the moment they were admitted to ICU with whose were not and, on the other hand, between those patients who received HAART during their ICU stay and those who not. 
Table 3. Comparison between patients who receive HAART and those who not (pre-ICU admission and during ICU stay).

\begin{tabular}{|c|c|c|c|c|c|c|}
\hline \multirow[t]{2}{*}{ Variable } & \multicolumn{3}{|c|}{ HAART pre-ICU admission } & \multicolumn{3}{|c|}{ HAART in the ICU } \\
\hline & $\begin{array}{c}\text { No } \\
(n=128)\end{array}$ & $\begin{array}{c}\text { Yes } \\
(n=155)\end{array}$ & $p$ & $\begin{array}{c}\text { No } \\
(n=172)\end{array}$ & $\begin{array}{c}\text { Yes } \\
(n=111)\end{array}$ & $p$ \\
\hline Age & $41.2 \pm 8.8$ & $45.2 \pm 9.9$ & $<0.01$ & $42.1 \pm 8.6$ & $45.4 \pm 10.7$ & $<0.01$ \\
\hline Male sex & $92(71.9)$ & $114(73.5)$ & 0.75 & $130(75.6)$ & $76(68.5)$ & 0.19 \\
\hline Admission from $E D$ & $73(57.0)$ & $87(56.1)$ & 0.88 & $96(55.8)$ & $64(57.7)$ & 0.76 \\
\hline \multicolumn{7}{|l|}{ HIV infection } \\
\hline HAART pre-ICU admission & & & & $70(45.2)$ & $85(54.8)$ & $<0.01$ \\
\hline HAART in the ICU & $26(20.3)$ & $85(54.8)$ & $<0.01$ & & & \\
\hline AIDS & $100(78.1)$ & $95(61.3)$ & $<0.01$ & $124(72.1)$ & $71(64.0)$ & 0.15 \\
\hline Years of infection & $6(0,12)$ & $12.5(6.25,18)$ & $<0.01$ & $9(2,16)$ & $10(3.75,17)$ & 0.34 \\
\hline CD4 count (cell//mL) & $72(30,182)$ & $293(130.25,518.25)$ & $<0.01$ & $160(53,317)$ & $185(54.75,518,25)$ & 0.12 \\
\hline Viral load (log) & $5.3(4.35,5.7)$ & $1.6(0,2.78)$ & $<0.01$ & $4.3(2,5.3)$ & $1.8(0,4.9)$ & $<0.01$ \\
\hline Viral load suppressed & $4(4.3)$ & $60(44.4)$ & $<0.01$ & $28(22.0)$ & $36(35.6)$ & 0.02 \\
\hline Days until start of HAART & $6(4,14)$ & $0(0,1)$ & $<0.01$ & & $1(0,5)$ & \\
\hline \multicolumn{7}{|c|}{ Comorbidities and nutritional status (see Appendix A. Supplementary data) } \\
\hline M-S liver disease & $12(9.4)$ & $25(16.1)$ & 0.09 & $27(15.7)$ & $10(9.0)$ & 0.10 \\
\hline Hepatitis C Virus (HCV) & 79 (61.7) & $91(58.7)$ & 0.61 & $118(68.6)$ & $52(46.8)$ & $<0.01$ \\
\hline Charlson Comorbidity Index & $7(6,7)$ & $6.5(2,8)$ & 0.48 & $7(3.25,8)$ & $6(2,7)$ & 0.08 \\
\hline Cachexia & $39(30.5)$ & $31(20.0)$ & 0.04 & $40(23.3)$ & $30(27.0)$ & 0.47 \\
\hline Albumin $(\mathrm{g} / \mathrm{dL})$ & $2.4(2,2.9)$ & $2.85(2.2,3.6)$ & $<0.01$ & $2.5(2.03,3.10)$ & $2.8(2.2,3,41)$ & $<0.01$ \\
\hline \multicolumn{7}{|c|}{ Diagnosis at admission (see Appendix A. Supplementary data) } \\
\hline Severe sepsis/septic shock & $81(63.3)$ & $59(38.1)$ & $<0.01$ & $87(50.6)$ & $53(47.7)$ & 0.64 \\
\hline PCP & $22(17.2)$ & $3(1.9)$ & $<0.01$ & $14(8.1)$ & $11(9.9)$ & 0.61 \\
\hline Cardiac & $10(7.8)$ & $45(29)$ & $<0.01$ & $24(14.0)$ & $31(27.9)$ & $<0.01$ \\
\hline \multicolumn{7}{|l|}{ Organ support during admission } \\
\hline Mechanical ventilation & $88(68.8)$ & $84(54.2)$ & 0.01 & $109(63.4)$ & $63(56.8)$ & 0.27 \\
\hline Vasoactive drugs & $56(43.8)$ & $58(37.4)$ & 0.28 & 75 (43.6) & $39(35.1)$ & 0.16 \\
\hline Renal replacement therapy & 15 (11.7) & $16(10.3)$ & 0.71 & $18(10.5)$ & $13(11.7)$ & 0.74 \\
\hline \multicolumn{7}{|c|}{ Complications (see Appendix A. Supplementary data) } \\
\hline Superinfection & $22(17.2)$ & $19(12.3)$ & 0.24 & $20(11.6)$ & $21(18.9)$ & 0.09 \\
\hline HAART-related* & $7(26.9)$ & $3(3.5)$ & $<0.01$ & & $10(9.0)$ & \\
\hline \multicolumn{7}{|l|}{ Severity scores } \\
\hline APACHE II & $20(13,26)$ & $15(10,23)$ & $<0.01$ & $19(13,26)$ & $15(9,23)$ & $<0.01$ \\
\hline SOFA score & $6(4,10)$ & $5(2,9)$ & 0.02 & $7(3.25,10)$ & $4(2,8)$ & $<0.01$ \\
\hline SOFA score $72 \mathrm{~h}$ & $5(2,8.25)$ & $4(2,8)$ & 0.16 & $5(2,9)$ & $4(1,7.5)$ & 0.21 \\
\hline \multicolumn{7}{|l|}{ Outcomes } \\
\hline ICU mortality & $35(27.3)$ & $36(23.2)$ & 0.42 & $53(30.8)$ & $18(16.2)$ & $<0.01$ \\
\hline Hospital mortality & $50(39.1)$ & $43(27.7)$ & 0.04 & $69(40.1)$ & 24 (21.6) & $<0.01$ \\
\hline ICU LOS & $6(2,15)$ & $4(2,10)$ & 0.04 & $4(1,9)$ & $8(3,17)$ & $<0.01$ \\
\hline Hospital LOS & $23.5(12.25,40.75)$ & $14(7,31)$ & $<0.01$ & $16.5(8,33,25)$ & $23(9,43)$ & 0.02 \\
\hline
\end{tabular}


We found HAART-related complications in less than $10 \%$ of patients who receive treatment during ICU stay (Appendix A. Supplementary data, Table e3), more frequently in patients without previous ART $(26.9$ vs $3.5 \%, p<0.01)$.

Factors significantly associated with hospital mortality on univariate analysis were included in the final regression model, and we found that only admission from a site other than the ED ((OR 3.64, 95\% CI 1.30-10.20, $p=0.01$ ), the existence of moderate-severe liver disease (OR 5.65, 95\% CI $1.11-28.87, p=0.04)$, APACHE II (OR $1.14,95 \%$ CI $1.04-1.26, p<0.01)$ and SOFA at $72 \mathrm{~h}(\mathrm{OR}$ $1.19,95 \%$ CI $1.02-1.40, p=0.03)$ maintained a statistically significant relationship with hospital mortality (Table 4).

Table 4. Variables related to hospital mortality. Univariate and multivariate logistic regression analysis.

\begin{tabular}{|c|c|c|c|c|c|c|}
\hline \multirow[b]{2}{*}{ Variable } & \multicolumn{3}{|c|}{$\begin{array}{l}\text { Univariate logistic regression } \\
\text { analysis }\end{array}$} & \multicolumn{3}{|c|}{$\begin{array}{l}\text { Multivariate logistic regression } \\
\text { analysis }\end{array}$} \\
\hline & OR & $95 \% \mathrm{CI}$ & $P$ value & OR & $95 \% \mathrm{CI}$ & $P$ value \\
\hline Male sex & 1.71 & $0.95-3.09$ & 0.08 & & & \\
\hline Age & 1.01 & $0.98-1.03$ & 0.73 & & & \\
\hline Admission not from ED & 3.94 & $2.33-6.65$ & $<0.01$ & 3.64 & $1.30-10.20$ & 0.01 \\
\hline \multicolumn{7}{|c|}{ Comorbidities and nutritional status } \\
\hline M-S liver disease & 2.45 & $1.22-4.94$ & 0.01 & 5.65 & $1.11-28.87$ & 0.04 \\
\hline Charlson Comorbidity Index & 1.11 & $1.02-1.22$ & 0.02 & 0.99 & $0.71-1.38$ & 0.94 \\
\hline $\begin{array}{l}\text { Cachexia on physical } \\
\text { examination }\end{array}$ & 2.67 & $1.53-4.67$ & $<0.01$ & 1.17 & $0.35-3.97$ & 0.80 \\
\hline Albumin (per g/dL) & 0.48 & $0.32-0.71$ & $<0.01$ & 0.87 & $0.33-2.32$ & 0.79 \\
\hline \multicolumn{7}{|l|}{ HIV infection } \\
\hline Years of infection & 0.98 & $0.94-1.01$ & 0.16 & & & \\
\hline $\begin{array}{l}\text { Diagnosis during actual } \\
\text { admission }\end{array}$ & 2.43 & $1.17-5.07$ & 0.02 & 1.55 & $0.32-7.56$ & 0.59 \\
\hline Not receiving HAART & 1.67 & $1.01-2.75$ & 0.04 & 1.49 & $0.44-5.11$ & 0.52 \\
\hline HAART during ICU stay & 0.41 & $0.24-0.71$ & $<0.01$ & 0.37 & $0.12-1.17$ & 0.09 \\
\hline AIDS & 1.72 & $0.98-3.02$ & 0.06 & & & \\
\hline CD4 count & 0.99 & $0.99-0.99$ & $<0.01$ & 0.99 & $0.99-1.00$ & 0.09 \\
\hline Viral load & 1.16 & $1.02-1.31$ & 0.02 & 1.11 & $0.83-1.49$ & 0.48 \\
\hline Viral load suppressed & 0.76 & $0.40-1.44$ & 0.40 & & & \\
\hline \multicolumn{7}{|l|}{ Diagnosis at admission } \\
\hline Severe sepsis/septic shock & 2.04 & $1.23-3.39$ & $<0.01$ & 0.65 & $0.18-2.37$ & 0.52 \\
\hline Cardiological & 0.28 & $0.12-0.65$ & $<0.01$ & 0.66 & $0.05-8.67$ & 0.76 \\
\hline Neurological & 3.79 & $1.08-13.27$ & 0.04 & 3.40 & $0.23-49.93$ & 0.37 \\
\hline Trauma & 0.52 & $0.17-1.63$ & 0.26 & & & \\
\hline \multicolumn{7}{|l|}{ Organ support during admission } \\
\hline Mechanical ventilation & 7.34 & $3.76-14.35$ & $<0.01$ & 2.90 & $0.53-15.97$ & 0.22 \\
\hline Vasoactive drugs & 4.26 & $2.52-7.21$ & $<0.01$ & 0.75 & $0.22-2.57$ & 0.64 \\
\hline Renal replacement therapy & 6.23 & $2.74-14.18$ & $<0.01$ & 2.22 & $0.57-8.63$ & 0.25 \\
\hline \multicolumn{7}{|l|}{ Complications } \\
\hline Superinfection & 2.79 & $1.42-5.47$ & $<0.01$ & 2.94 & $0.91-9.43$ & 0.07 \\
\hline \multicolumn{7}{|l|}{ Severity scores } \\
\hline APACHE II & 1.16 & $1.11-1.20$ & $<0.01$ & 1.14 & $1.04-1.26$ & $<0.01$ \\
\hline SOFA score & 1.24 & $1.16-1.33$ & $<0.01$ & 0.80 & $0.64-1.00$ & 0.06 \\
\hline SOFA score $72 \mathrm{~h}$ & 1.22 & $1.13-1.32$ & $<0.01$ & 1.19 & $1.02-1.40$ & 0.03 \\
\hline
\end{tabular}

ED: Emergency department; HAART: high-activity antiretroviral therapy; OR: odds ratio; CI: confidence interval. 


\section{Discussion}

Our study describes the HIV-infected patient population that required ICU admission over a relatively long period (15 years), and during the current period of ART. Consistent with most publications to date, we found that critical patients infected with HIV are young and predominantly male. In our study, we could not prove a relation between immunovirological or nutritional status, reason for admission or need for organ support and hospital mortality. Variables related with higher hospital mortality in our patients were severity scores (APACHE II at admission and SOFA at $72 \mathrm{~h}$ ), moderate-severe liver disease and admission form hospital ward. Our data provide important insight regarding the complex management of hospitalised HIVinfected patients. Sepsis remains the leading cause of ICU admission in the current HAART era. Mortality, although higher, increasingly approximates that of non-HIV patients.

Our population has a long-standing history of clinical course of infection (median nine years from diagnosis, increasing up to 12 in last five years of our study) and very few patients were diagnosed during their current admission (less than 12\%). These data are similar to those recently published describing industrialized countries. ${ }^{22,28,33}$ However, in the studies by Chiang et al. and Xiao et al., the clinical course of the disease was much shorter (less than a year), with a high percentage of diagnoses during admission (approximately 50\%). ${ }^{26,29}$

At present, it is widely currently recommended that HAART be administered to all HIV-infected patients, regardless of immunovirological profile or symptoms. However, despite that proportion of patients receiving HAART is increasing, only $54.8 \%$ of our patients were under HAART at admission. These data are comparable to those reported by other countries from our region, ${ }^{18,28,33}$ and the level in Spain is higher than in the studies published from countries such as Australia, Brazil, Taiwan and China (in which less than $40 \%$ of patients received ART), ${ }^{22,26,27,29}$ but far from the $71 \%$ reported in the USA by Akgün et al. ${ }^{20}$ This variability in the percentage of patients who receive ART is reflected in their CD4 T-lymphocyte counts at admission. Among our patients, the median was $167 \mathrm{cells} / \mathrm{mm}^{3}(53.5,414.0)$; in studies with a lower percentage of patients receiving ART, the CD4 count is lower, even falling below 50 cells $/ \mathrm{mm}^{3}{ }^{26,29}$

As in other recent studies, despite our patients' relatively good immunovirological conditions, almost half were admitted to the ICU because of infection. ${ }^{22,23}$

Consistent with previous reports, ${ }^{22,31} 13.1 \%$ of our patients presented with chronic liver disease with cirrhosis or PHT. HCV co-infection is very common in HIV patients and varies between $15 \%$ and $60 \%^{19,31}$; in our sample, $60.1 \%$ of patients presented with HCV co-infection.

ICU and hospital mortality $(25.1 \%$ and $32.9 \%$, respectively) observed in our study was comparable to the figures reported for other industrialized countries $^{6,7,8,9,10,11,12,13,14,15,16,17,18,19,20,21,22,23}$ and lower than those reported for developing countries (higher than $40 \%)^{24,25,26,27,28,29,30}$ and in other Spanish studies. ${ }^{31,32}$ These observed mortality differences are probably of multifactorial origin and impact both factors such as extrahospital treatment for these patients (follow-up in consultations, HAART, management of comorbidities, etc.) and the care received during hospital admission (ICU admission criteria, organ support provided, etc.).

We did not find a significant relationship between immunovirological condition, receiving ART, or years of evolution of infection and hospital mortality. These data coincide with those reported in most, ${ }^{8}, 10,15,16,18,19,22,26,27,29,33,36,37$ although not all, ${ }^{11}, 13,17,22,24,26,28,38$ studies published to date. Probably the difficulty in detecting an independent relationship between mortality and inmunovorological status lies in the fact that patients who receive HAART have not only a higher CD4 count and a lower VL but also a better nutritional status and a different reason for ICU 
admission. Nevertheless, a larger sample size could, perhaps, give us a statistically significant relation of CD count with mortality.

Furthermore, some studies have related the use of HAART during the ICU stay to lower intra-ICU mortality without impacting long-term survival. ${ }^{19,25,27,33}$ A meta-analysis including 12 retrospectives studies, published in 2017, showed a significant benefit on short-term mortality when HAART is received during ICU stay, but long-term benefit remains unclear. ${ }^{39}$ Herein, despite we found a lower hospital mortality in patients having HAART both previously and during ICU stay, we could not prove a significant relationship between receiving HAART and hospital mortality. Although is possible that inclusion of a higher number of patients would change this, there were important differences between these groups. On the one hand, patients under HAART at admission had a better immunovirological and nutritional status, less ICU admissions caused by sepsis and lower severity scores at admission. On the other hand, severity scores were higher in patients who did not receive HAART during ICU stay and patients who receive HAART on ICU were more frequently under treatment at admission (with a consequent better immunovirological status). It's remarkable that only $54 \%$ patients with chronic HAART treatment receive it during ICU stay. HAART administration in ICU is complicated due to limited availability of parenteral ART and unknown pharmacokinetics of enteral administration through nasogastric tubes in patients receiving mechanical ventilation or in shock, risk of potential drugs interactions and risk of provoking immune reconstitution inflammatory syndrome. The delay in the start of HAART in ICU was $1(0,5)$ day, but was higher in patients with invasive mechanical ventilation $[4.5(1,7)$ compared with $0(0,1)$ days in no-ventilated patients, $p<0.01]$ or in shock $[5(1,8.5)$ vs $1(0,3)$ days].

Just as in other studies, ${ }^{21,27,28,29,30}$ delayed ICU admission from patient hospital arrival (here defined as admission not from E.D.) significantly increased the risk of death during the hospital stay. Factors such as initially inadequate treatment, poor response to that treatment, or onset of complications during the hospital stay may justify the relationship between delayed admission and higher mortality.

In the current era, in which HIV infection is controllable with ART, comorbidities play an increasingly important role in patient prognosis. Patients with a history of moderate-severe liver disease have a higher risk of hospital death in our study and those by Barbier et al., Turvey et al. and Medrano et al. ${ }^{21,22,31} \mathrm{We}$ did not find relation between other comorbidities and mortality.

It has been proposed that, nowadays, the mortality among ICU-admitted HIV patients is related to the severity of their acute event. Higher severity scores among critical patients at admission, such as APACHE II or SAPS II, have been related to higher mortality. ${ }^{19,21,22,25,27,28,33}$ This was observed in our study, in which risk of death increases close to $20 \%$ for each point on SOFA score evaluated at $72 \mathrm{~h}$ and more than $10 \%$ for each point on the APACHE II score. However, we did not find a significant relationship between the SOFA at ICU admission and mortality, which probably reflects that in order to establish the prognosis it is more important to assess the response to treatment than the initial organ dysfunction. Regarding the need for organ support, evidence is contradictory, with discrepancies as to the relationship between hospital mortality and the need for respiratory support with invasive MV,,$^{19,21,22,25,27,29}$ for VADs ${ }^{19,21,22}$ and for RRTs. ${ }^{21,22,29}$ In our study, the need for support did not predict hospital mortality once we adjusted for severity, reason for admission and comorbidities.

While some studies have reported poorer prognosis in patients admitted to the ICU because of infection, ${ }^{25,30}$ we did not detect any relationship between the reason for ICU admission and prognosis. These differences may be based on patients' immunovirological profiles at admission, because more immunosuppression increases their risk of admission due to OIs, which then lead to a worse prognosis. 
Finally, we found important differences between the three periods (2000-2004, 2005-2009 and 2010-2014). First, patients were older and with more years living with HIV in most recent periods. Second: proportion of patients receiving HAART also increases significantly, with a consequent decrease in HIV viral load. Third, severity scores were higher in most recent periods. We did not find changes in mortality over time (Table 2).

Our study is not without limitations. First, it was observational and retrospective. Second, although two sites took part, both are in the same geographic setting, limiting the extent to which we can generalize the results. Third, data on ART administration were obtained from clinical records; no drug level tests were performed either at admission or during the ICU stay. Thus, it is possible that patients noncompliant with treatment were not detected. Fourth, only physical examination and albumin levels at admission were used to evaluate nutritional status; we do not have other useful data such as body mass index. Fifth, a recruitment bias cannot be ruled out, because neither of the two units taking part have defined ICU admission criteria for HIV patients; we identified patients retrospectively using the hospital records system. Finally, our study includes patients over 15 years, which may reduce its external validity.

\section{Conclusions}

Delayed ICU admission, comorbidities and severity of critical illness, determine the prognosis of HIV-infected patients admitted to the ICU. We did not detect any impact of immunovirological or nutritional condition, reason for admission, or need for organ support during admission. Based on these data, HIV patients should receive the same level of care as non-HIV-infected patients, regardless of their immunological or nutritional condition. We can not prove a relationship between receiving HAART and prognosis, but we have shown significant differences between patients who receive and do not receive treatment before ICU admission (better nutritional and inmunovirologic status, and less severity, sepsis-related admissions and need for mechanical ventilation in HAART patients) and during their ICU stay (better immunological status, less HCVinfected patients and less severity and more cardiac-related diagnosis in patients who received HAART).

\section{Autorship}

Pablo Vidal-Cortés has participated in the conception and design of the study and the acquisition, analysis and interpretation of the data and has made the draft of the article.

Luis Antonio Álvarez-Rocha has participated in the conception and design of the study, and in the critical review of intellectual content. He has approved the version that is presented.

Paula Fernández-Ugidos has participated in the acquisition of data and has approved the version presented.

Marcos Antonio Pérez-Veloso has participated in the acquisition of data and has approved the version presented.

Ignacio María Suárez-Paul has participated in the acquisition of data and has approved the version presented.

Alejandra Virgós-Pedreira has participated in the acquisition of data and has approved the version presented.

Sonia Pértega-Díaz has participated in the analysis and interpretation of data, in the critical review of intellectual content, and has approved the version presented.

Ángeles Castro-Iglesias has participated in the conception and design of the study, in the critical review of the intellectual content, and has approved the version presented. 


\section{Conflict of interests}

The authors declare no conflict of interest.

\section{Acknowledgments}

To the memory of Dr. Salvador Pita, who contributed to the design of the study and the analysis of the data, with great gratitude from the authors.

\section{References}

1. Collaboration ATC. Life expectancy of individuals on combination antiretroviral therapy in highincome countries: a collaborative analysis of 14 cohort studies. Lancet. 2008;372:293-9.

2. van Sighem AI, Gras LA, Reiss P, Brinkman K, de Wolf F, study Anoc. Life expectancy of recently diagnosed asymptomatic HIVinfected patients approaches that of uninfected individuals. AIDS. 2010;24:1527-35.

3. Boulle A, Schomaker M, May MT, Hogg RS, Shepherd BE, Monge S, et al. Mortality in patients with HIV-1 infection starting antiretroviral therapy in South Africa Europe, or North America: a collaborative analysis of prospective studies. PLoS Med. 2014;11:e1001718.

4. Berry SA, Fleishman JA, Moore RD, Gebo KA, Network HR. Trends in reasons for hospitalization in a multisite United States cohort of persons living with HIV, 2001-2008. J Acquir Immune Defic Syndr. 2012;59:368-75.

5. Deeks SG, Lewin SR, Havlir DV. The end of AIDS: HIV infection as a chronic disease. Lancet. 2013;382:1525-33.

6. Nickas G, Wachter RM. Outcomes of intensive care for patients with human immunodeficiency virus infection. Arch Intern Med. 2000;160:541-7.

7. Afessa B, Green B. Clinical course, prognostic factors, and outcome prediction for HIV patients in the ICU. The PIP (Pulmonary complications, ICU support, and prognostic factors in hospitalized patients with HIV) study. Chest. 2000;118:138-45.

8. Morris A, Creasman J, Turner J, Luce JM, Wachter RM, Huang L. Intensive care of human immunodeficiency virus-infected patients during the era of highly active antiretroviral therapy. Am J Respir Crit Care Med. 2002;166:262-7.

9. Nuesch R, Geigy N, Schaedler E, Battegay M. Effect of highly active antiretroviral therapy on hospitalization characteristics of HIV-infected patients. Eur J Clin Microbiol Infect Dis. 2002;21:684-7.

10. Narasimhan M, Posner AJ, DePalo VA, Mayo PH, Rosen MJ. Intensive care in patients with HIV infection in the era of highly active antiretroviral therapy. Chest. 2004;125:1800-4.

11. Vincent B, Timsit JF, Auburtin M, Schortgen F, Bouadma L, Wolff M, et al. Characteristics and outcomes of HIV-infected patients in the ICU: impact of the highly active antiretroviral treatment era. Intensive Care Med. 2004;30:859-66.

12. Casalino E, Wolff M, Ravaud P, Choquet C, Bruneel F, Regnier B. Impact of HAART advent on admission patterns and survival in HIV-infected patients admitted to an intensive care unit. AIDS. 2004;18:1429-33.

13. Dickson SJ, Batson S, Copas AJ, Edwards SG, Singer M, Miller RF. Survival of HIV-infected patients in the intensive care unit in the era of highly active antiretroviral therapy. Thorax. 2007;62:964-8.

14. Powell K, Davis JL, Morris AM, Chi A, Bensley MR, Huang L. Survival for patients With HIV admitted to the ICU continues to improve in the current era of combination antiretroviral therapy. Chest. 2009;135:11-7.

15. Coquet I, Pavie J, Palmer P, Barbier F, Legriel S, Mayaux J, et al. Survival trends in critically ill HIVinfected patients in the highly active antiretroviral therapy era. Crit Care. 2010;14:R107.

16. van Lelyveld SF, Wind CM, Mudrikova T, van Leeuwen HJ, de Lange DW, Hoepelman AI. Short- and long-term outcome of HIVinfected patients admitted to the intensive care unit. Eur J Clin Microbiol Infect Dis. 2011;30:1085-93.

17. Adlakha A, Pavlou M, Walker DA, Copas AJ, Dufty N, Batson S, et al. Survival of HIV-infected patients admitted to the intensive care unit in the era of highly active antiretroviral therapy. Int J STD AIDS. 2011;22:498-504.

18. Turtle L, Vyakernam R, Menon-Johansson A, Nelson MR, Soni N. Intensive care usage by HIVpositive patients in the HAART era. Interdiscip Perspect Infect Dis. 2011;2011:847835. 
19. Morquin D, Le Moing V, Mura T, Makinson A, Klouche K, Jonquet O, et al. Short- and long-term outcomes of HIV-infected patients admitted to the intensive care unit: impact of antiretroviral therapy and immunovirological status. Ann Intensive Care. 2012;2:25.

20. Akgün KM, Tate JP, Pisani M, Fried T, Butt AA, Gibert CL, et al. Medical ICU admission diagnoses and outcomes in human immunodeficiency virus-infected and virus-uninfected veterans in the combination antiretroviral era. Crit Care Med. 2013;41:1458-67.

21. Barbier F, Roux A, Canet E, Martel-Samb P, Aegerter P, Wolff M, et al. Temporal trends in critical events complicating HIV infection: 1999---2010 multicentre cohort study in France. Intensive Care Med. 2014;40:1906-15.

22. Turvey SL, Bagshaw SM, Eurich DT, Sligl WI. Epidemiology and outcomes in critically ill patients with human immunodeficiency virus infection in the era of combination antiretroviral therapy. Can J Infect Dis Med Microbiol. 2017;2017:7868954.

23. Huson MA, Bakhshi-Raiez F, Grobusch MP, de Jonge E, de Keizer NF, van der Poll T. Characteristics and outcome of patients with AIDS in Dutch ICUs between 1997 and 2014. Crit Care Med. 2016;44:291-9.

24. Vargas-Infante YA, Guerrero ML, Ruiz-Palacios GM, Soto-Ramírez LE, Del Río C, Carranza J, et al. Improving outcome of human immunodeficiency virus-infected patients in a Mexican intensive care unit. Arch Med Res. 2007;38:827-33.

25. Croda J, Croda MG, Neves A, De Sousa dos Santos S. Benefit of antiretroviral therapy on survival of human immunodeficiency virus-infected patients admitted to an intensive care unit. Crit Care Med. 2009;37:1605-11.

26. Chiang HH, Hung CC, Lee CM, Chen HY, Chen MY, Sheng WH, et al. Admissions to intensive care unit of HIV-infected patients in the era of highly active antiretroviral therapy: etiology and prognostic factors. Crit Care. 2011;15:R202.

27. Amâncio FF, Lambertucci JR, Cota GF, Antunes CM. Predictors of the short- and long-term survival of HIV-infected patients admitted to a Brazilian intensive care unit. Int J STD AIDS. 2012;23:692-7.

28. Foo H, Clezy K, Post JJ. The long-term outcome of HIVinfected patients after intensive care admission. Int J STD AIDS. 2012;23:e4-8.

29. Xiao J, Zhang W, Huang Y, Tian Y, Su W, Li Y, et al. Etiology and outcomes for patients infected with HIV in intensive care units in a tertiary care hospital in China. J Med Virol. 2015;87:366-74.

30. Japiassú AM, Amâncio RT, Mesquita EC, Medeiros DM, Bernal HB, Nunes EP, et al. Sepsis is a major determinant of outcome in critically ill HIV/AIDS patients. Crit Care. 2010;14:R152.

31. Medrano J, Álvaro-Meca A, Boyer A, Jiménez-Sousa MA, Resino S. Mortality of patients infected with HIV in the intensive care unit (2005 through 2010): significant role of chronic hepatitis $\mathrm{C}$ and severe sepsis. Crit Care. 2014;18:475.

32. Palacios R, Hidalgo A, Reina C, de la Torre M, Márquez M, Santos J. Effect of antiretroviral therapy on admissions of HIV-infected patients to an intensive care unit. HIV Med. 2006;7:193-6.

33. Meybeck A, Lecomte L, Valette M, Van Grunderbeeck N, Boussekey N, Chiche A, et al. Should highly active antiretroviral therapy be prescribed in critically ill HIV-infected patients during the ICU stay? A retrospective cohort study. AIDS Res Ther. 2012;9:27.

34. 1993 revised classification system for HIV infection and expanded surveillance case definition for AIDS among adolescents and adults. MMWR Recomm Rep. 1992;41:1-19.

35. (CDC) CfDCaP. Revised surveillance case definition for HIV infection - United States, 2014. MMWR Recomm Rep. 2014;63:1-10.

36. Afessa B, Green B. Bacterial pneumonia in hospitalized patients with HIV infection: the Pulmonary Complications ICU Support, and Prognostic Factors of Hospitalized Patients with HIV (PIP) Study. Chest. 2000;117:1017-22.

37. Rosen MJ, Clayton K, Schneider RF, Fulkerson W, Rao AV, Stansell J, et al. Intensive care of patients with HIV infection: utilization, critical illnesses, and outcomes. Pulmonary Complications of HIV Infection. Am J Respir Crit Care Med. 1997;155:67-71.

38. Khouli H, Afrasiabi A, Shibli M, Hajal R, Barrett CR, Homel P. Outcome of critically ill human immunodeficiency virus-infected patients in the era of highly active antiretroviral therapy. J Intensive Care Med. 2005;20:327-33.

39. Andrade HB, Shinotsuka CR, da Silva IRF, Donini CS, Yeh Li H, de Carvalho FB, et al. Highly active antiretroviral therapy for critically ill HIV patients: A systematic review and meta-analysis. PLOS ONE. 2017;12:e0186968. 